\title{
Random Domain Decomposition for Flow in Heterogeneous Stratified Aquifers
}

\author{
Alberto Guadagnini $^{(1)}$, Laura Guadagnini ${ }^{(1)}$, Daniel M. Tartakovsky ${ }^{(2)}$ and C.L. Winter ${ }^{(3)}$ \\ (1) Dipartimento Ingegneria Idraulica, Ambientale, Infrastrutture Viarie, Rilevamento \\ (D.I.I.A.R.), Politecnico di Milano, Piazza L. Da Vinci, 32, 20133 Milano, Italy. E-mail: \\ alberto.guadagnini@polimi.it and laura.guadagnini@polimi.it \\ (2) Theoretical Division; Los Alamos National Laboratory; Los Alamos, USA. E-mail: \\ dmt@lanl.gov \\ (3) National Center for Atmospheric Research, 1850 Table Mesa Drive, Boulder, CO 80305. \\ E-mail: 1winter@ucar.edu
}

\begin{abstract}
We study two-dimensional flow in a layered heterogeneous medium composed of two materials whose hydraulic properties and spatial distribution are known statistically but are otherwise uncertain. Our analysis relies on the composite media theory, which employs random domain decomposition in the context of groundwater flow moment equations to explicitly account for the separate effects of material and geometric uncertainty on ensemble moments of head and flux. Flow parallel and perpendicular to the layering in a two-material composite layered medium is considered. The hydraulic conductivity of each material is lognormally distributed with a much higher mean in one material than in the other. The hydraulic conductivities of points within different materials are uncorrelated. The location of the internal boundary between the two contrasting materials is random and normally distributed with given mean and variance. We solve the equations for (ensemble) moments of hydraulic head and flux and analyze the impact of unknown geometry of materials on statistical moments of head and flux. We compare the composite media approach to approximations that replace statistically inhomogeneous conductivity fields with pseudo-homogeneous random fields.
\end{abstract}

\section{Introduction}

It has long been recognized that deterministic analyses of flow and transport in the subsurface environment are rendered less than optimal by the lack of detailed site characterization of the kind required for most high-resolution numerical simulations. Uncertainty in hydraulic and transport parameters of natural geologic formations is conveniently accounted for by treating them as random fields. Consequently, flow and transport equations become stochastic.

Much of the existing literature on stochastic hydrogeology deals with mildly heterogeneous formations, where variance of log-hydraulic conductivity, $\sigma_{Y}^{2}$, is relatively small. This assumption is crucial for closing the moment differential equations or for making Monte Carlo simulations manageable. At the same time, it clearly limits the applicability of most such analyses. Several approaches have emerged to deal with highly heterogeneous natural formations composed of multiple geological facies. For an in-depth review of the current state-of-the-art we refer the interested reader to Winter et al. [2003], while providing a brief outline here.

Two models of heterogeneity have been intensively investigated in the literature. An early method of modelling flow and transport in composite aquifers (i.e., aquifers composed of multiple geological facies) relies on removing deterministic trends in hydraulic conductivity [e.g., McLaughlin and Wood, 1988]. De-trending techniques assume that the conductivity residuals are statistically homogeneous and that the geometries of geological facies are known with certainty. In particular, models with deterministic trends require 
hydraulic conductivity to have the same correlation structure within each geological facies and between facies. When the spatial arrangement of geological facies is unknown, hydraulic conductivity can be treated as a random field with multi-modal distributions [e.g., Desbarats, 1990]. Models with multi-modal distributions result in large variance of log-hydraulic conductivity when geological facies are sufficiently different, which renders perturbation expansions unworkable. We call these models homogeneous approximations to the heterogeneous system.

The recently proposed method of random domain decomposition [Winter and Tartakovsky, 2000, 2002; and Winter et al., 2002] provides a more general framework for modelling flow and transport in heterogeneous composite porous media. It allows for uncertainty in both spatial arrangement of geological facies and hydraulic properties within each facies. Because perturbation expansions are carried out within each facies separately, their accuracy and robustness remain high for most geological settings. In what follows we employ random domain decomposition (RDD) to analyze two-dimensional steady-state flow in layered aquifers with uncertain boundaries between layers and hydraulic conductivities within each layer. Layered media are of practical interest due to their simplicity and because they provide an example of structural heterogeneity which arises in natural sedimentary formations. While perfect layering represents an idealization of a sedimentary medium, the composite medium scheme allows taking into account its likely imperfect description. This renders the model more appealing to applications over short distances and to grasp the key aspects of the flow processes. The main goals of this study are to verify the accuracy of the flow statistics obtained by RDD and to evaluate the relative impact of the two sources of uncertainty in such a setting.

We start by formulating the problem of flow in heterogeneous layered aquifers and by outlining the RDD approach in Section 2. Accuracy of the perturbation approximations for the first two statistical moments of hydraulic head (Section 3) and Darcy flux (Section 4) is ascertained via comparison with Monte Carlo simulations. In Sections 3 and 4 we also analyze the relative importance of uncertain geometry and uncertain conductivity by focusing on two special cases in which (1) the layer geometry is random, but the hydraulic properties of each material are fixed, and (2) the layer geometry is fixed but material properties vary. Finally, we compare the composite medium model to models with deterministic trends.

\section{Problem statement and RDD methodology}

Consider steady-state flow equation, $\nabla \cdot K \nabla h=f$, where $K$ is (random) hydraulic conductivity, $h$ is (random) hydraulic head and $f$ is the (random) source function. It is common to use the Reynolds decomposition to represent random fields $\mathfrak{R}=\langle\mathfrak{R}\rangle+\mathfrak{R}^{\prime}$ as the sum of their ensemble means $\langle\mathfrak{R}\rangle$ and zero-mean fluctuations $\mathfrak{R}^{\prime}$. Then averaging the stochastic flow equations gives

$\nabla \cdot[\langle K\rangle \nabla\langle h\rangle]-\nabla \cdot \mathbf{r}=\langle f\rangle$

where $\mathbf{r}=-\left\langle K^{\prime} \nabla h^{\prime}\right\rangle$ is the residual flux, representing the cross-covariance between hydraulic head gradient and conductivity fluctuations. Deriving approximations for the residual flux is the crucial part of any stochastic analysis. One of the most popular approaches is to use perturbation expansion in $\sigma_{Y}^{2}$, variance of log-hydraulic conductivity, $Y=\ln K$. Theoretically this limits applicability of such solutions to mildly heterogeneous aquifers or to highly heterogeneous aquifers, in the presence of a large number of hydraulic conductivity measurements. However, numerical simulations of Guadagnini and Neuman [1999b] demonstrated that first-order perturbation approximations of hydraulic heads and fluxes remain robust for moderately heterogeneous media with $\sigma_{Y}^{2}$ as large as 4 . Conditioning on conductivity data increases the applicability of such solutions to even higher variances. 
Random Domain Decomposition further extends the range of applicability of perturbation closures [Winter and Tartakovsky, 2000, 2002]. RDD recognizes that high spatial variability usually stems from the presence of different geological facies and explicitly accounts for it. As demonstrated by Winter et al. [2002], RDD renders perturbation approximations of the hydraulic head moments accurate and robust for $\sigma_{Y}^{2}$ as large as 20 . RDD treats the porous medium (and its conductivity) as a doubly stochastic process, where both facies geometries and their hydraulic conductivities are random. This allows one to obtain the statistics of hydraulic head and Darcy flux in two steps. The first step consists of calculating the conditional statistics of the system states via perturbation approximations, e.g.,

$\left\langle h^{[1]}(\boldsymbol{x} \mid b)\right\rangle=\left\langle h^{(0)}(\boldsymbol{x} \mid b)\right\rangle+\left\langle h^{(1)}(\boldsymbol{x} \mid b)\right\rangle$,

in powers of $\sigma_{Y_{M i}}^{2}$, the variance of log-conductivity within the facies $M$. Here superscript $(i)$ denotes terms in the expansion proportional to the $i$-th power of $\sigma_{Y_{M i}}^{2}$ and the vertical bar means conditioning on the facies geometry, such as the layer's width $b$ (Figure 1).

In a flow domain, $\Omega=\Omega_{1}+\Omega_{2}$, composed of two disjoint units, $\Omega_{1}$ and $\Omega_{2}$, with an uncertain boundary between them, deterministic hydraulic head, $H_{i}(i=1,2)$, prescribed on Dirichlet boundaries, $\Gamma_{D_{i}}$, and no flow Neumann boundaries, the zeroth- and first-order approximations of conditional mean hydraulic head in (2) are respectively given by

$\left\langle h^{(0)}(\boldsymbol{x} \mid b)\right\rangle=\sum_{i=1}^{2} \int_{\Omega_{i}}\langle f(\boldsymbol{y})\rangle G(\boldsymbol{y}, \boldsymbol{x}) d \boldsymbol{y}-\sum_{i=1}^{2} \int_{\Gamma_{D_{i}}} H_{i}(\boldsymbol{y}) K_{g_{i}} \boldsymbol{n}(\boldsymbol{y}) \cdot \nabla_{y} G(\boldsymbol{y}, \boldsymbol{x}) d \boldsymbol{y}$

$\left\langle h^{(1)}(\boldsymbol{x} \mid b)\right\rangle=\sum_{i=1}^{2} \int_{\Omega_{i}} \nabla_{y} G(\boldsymbol{y}, \boldsymbol{x}) \cdot\left[\boldsymbol{r}_{i}^{(1)}(\boldsymbol{y} \mid b)-K_{g_{i}} \frac{\sigma_{Y_{M i}}^{2}}{2} \nabla_{y}\left\langle h^{(0)}(\boldsymbol{y} \mid b)\right\rangle\right] d \boldsymbol{y}$

where $\boldsymbol{n}$ is the unit vector normal to the Dirichlet boundary, $K_{g i}$ is the geometric mean of the $i$ th conductivity $(i=1,2)$ and $G$ is the Green's function corresponding to the fixed boundary $b$ and $K_{g i}$. When hydraulic conductivities of the blocks $\Omega_{1}$ and $\Omega_{2}$ are uncorrelated, the firstorder approximation of the conditional mean residual flux, $\boldsymbol{r}_{i}^{(1)}(\boldsymbol{x} \mid b)$, is

$\boldsymbol{r}_{i}^{(1)}(\boldsymbol{x} \mid b)=K_{g_{i}}^{2} \int_{\Omega_{i}} C_{Y_{i}}(\boldsymbol{x}, \boldsymbol{y}) \nabla_{y} \nabla_{x}^{T} G(\boldsymbol{y}, \boldsymbol{x}) \nabla_{y}\left\langle h^{(0)}(\boldsymbol{y} \mid b)\right\rangle d \boldsymbol{y}$

See Winter and Tartakovky [2002] and Winter et al. [2002] for additional details.

The second step consists of calculating the corresponding statistics of the system states averaging over the geometry distribution $p(b)$, e.g.,

$\left\langle h^{[1]}(\boldsymbol{x})\right\rangle=\int\left\langle h^{[1]}(\boldsymbol{x} \mid b)\right\rangle p(b) d b$.

The conditional moment equations are solved numerically by finite elements program of Guadagnini and Neuman [1999a] and (6) is approximated by the law of large numbers,

$\left\langle h^{[1]}(\boldsymbol{x})\right\rangle \approx \frac{1}{n_{b}} \sum_{n=1}^{n_{b}}\left\langle h^{[1]}(\boldsymbol{x} \mid b)\right\rangle^{[1]}$

where $n_{b}$ is the number of realizations of $b$. Second-order statistics (variances) are calculated in a similar manner, 


$$
\left[\sigma_{h}^{2}(\boldsymbol{x})\right]^{[1]} \approx \frac{1}{n_{b}} \sum_{n=1}^{n_{b}}\left[\sigma_{h}^{2}(\boldsymbol{x} \mid b)\right]^{[1]}
$$

with the first-order approximation of conditional head variance $\left[\sigma_{h}^{2}(\boldsymbol{x} \mid b)\right]^{[1]}$ given by

$$
\left[\sigma_{h}^{2}(\boldsymbol{x} \mid b)\right]^{[1]}=-\sum_{i=1}^{2} \int_{\Omega_{i}} C_{K_{i} h}^{(1)}(\boldsymbol{y}, \boldsymbol{x} \mid b) \nabla_{y}\left\langle h^{(0)}(\boldsymbol{y} \mid b)\right\rangle \cdot \nabla_{y} G(\boldsymbol{y}, \boldsymbol{x}) d \boldsymbol{y}
$$

where the first-order approximation of the cross covariance $C_{K_{i} h}^{(1)}(\boldsymbol{y}, \boldsymbol{x})=\left\langle K_{i}^{\prime}(\boldsymbol{y}) h^{\prime}(\boldsymbol{x})\right\rangle$ is found as

$$
C_{K_{i} h}^{(1)}(\boldsymbol{y}, \boldsymbol{x} \mid b)=-K_{g_{i}}^{2} \int_{\Omega_{i}} C_{Y_{i}}(\boldsymbol{y}, \boldsymbol{z}) \nabla_{z}\left\langle h^{(0)}(\boldsymbol{z} \mid b)\right\rangle \cdot \nabla_{z} G(\boldsymbol{z}, \boldsymbol{x}) d \boldsymbol{z}
$$

Statistics for the flow can be derived in a similar manner.

To be specific we consider flow in a square domain composed of two layers of different materials separated by an uncertain boundary. The materials are characterized by hydraulic conductivities $K_{1}<<K_{2}$, which are treated as statistically homogeneous lognormally distributed random fields $Y_{i}=\ln K_{i},(i=1,2)$ with corresponding means, $\left\langle Y_{1}\right\rangle=3.5$ and $\left\langle Y_{2}\right\rangle=7.0$, variances, $\sigma_{Y_{M i}}^{2}=1$, and exponential correlation functions, $\rho_{Y_{i}}$, with unit correlation lengths $\lambda$. For simplicity, conductivities of different materials are taken to be uncorrelated. Of course, the resulting conductivity field is statistically inhomogeneous, in that its mean, variance and correlation function are all space dependent.

We impose constant heads, $\mathrm{H}_{\mathrm{A}}=1.6$ and $\mathrm{H}_{\mathrm{B}}=1.0$ (in arbitrary consistent units), on the vertical sides of a square domain of side $a=12 \lambda$ while assuming that the other two sides are impermeable. We consider two flow scenarios, (A) parallel and (B) perpendicular to the layering. A sketch of the two mean flow scenarios considered is presented in Figure 1. The domain is discretized by a grid of 3600 square elements ( 60 rows and 60 columns) of uniform size with 5 points per correlation length of $Y$.

On such domains we generated 5000 realizations of the location, $b$, of the internal boundary between the two contrasting materials upon taking it as a normally distributed random variable with given mean $\langle b\rangle=a / 2$ and variance, $\sigma_{b}^{2}$. We analyze the effect of uncertainty in the location of the layering on hydraulic head variance by considering $\sigma_{b}^{2}=0$, $0.1,0.5,2,3$. For each realization of $b$ we generated 3000 realizations of each of the two random materials on the grid spanning the whole square. Conditional realizations of our composite media were obtained by composing the two generated materials to create the desired layered domains. Guadagnini and Neuman [1999a, 1999b] noted that a complete stabilization of the Monte Carlo statistics is not necessary for a comparison between the solutions obtained from moment equations and from Monte Carlo simulations to be meaningful. Therefore we limit the number of our Monte Carlo simulations to 5000 for $b$ and 2000 for each of $Y_{\mathrm{i}}$. Since these 5000 realizations of $b$ fell within 60 discrete classes, due to the prescribed cell size, we performed a total of $60 \times 3000=180,000$ Monte Carlo simulations of the flow equations. We also considered the effect of models with a deterministic linear trend between the conductivities of the two contrasting materials. 

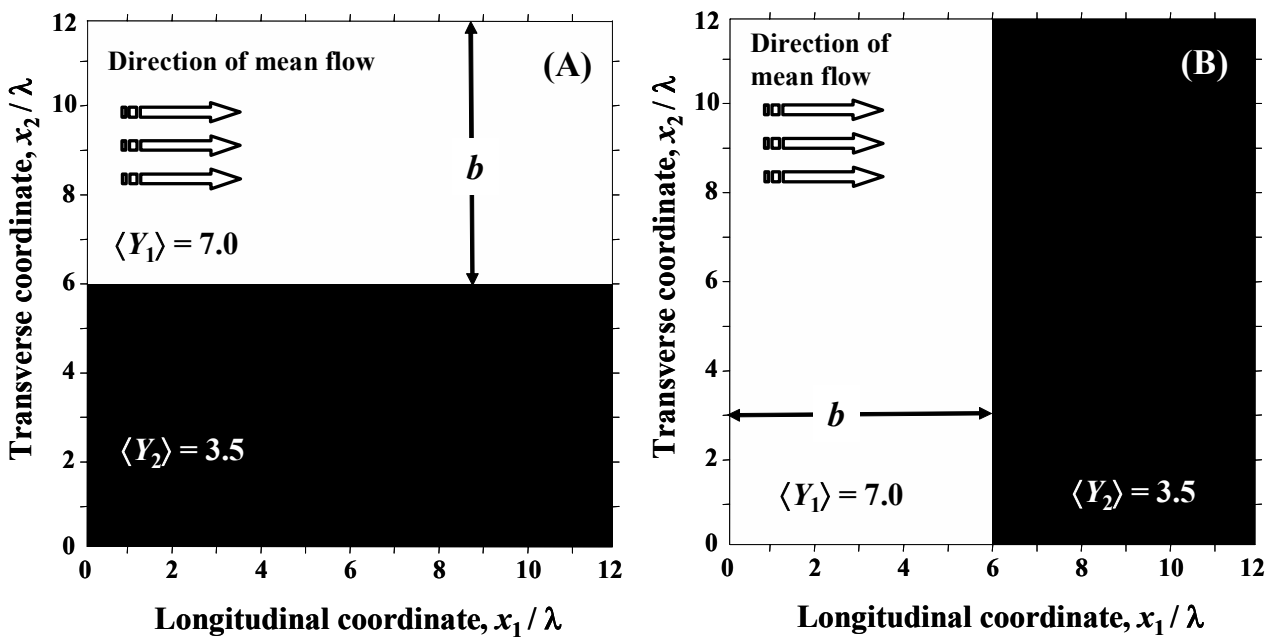

Figure 1. Flow domain for mean flow (A) parallel and (B) normal to layering.

By way of example, Figure 2 depicts the comparison between the total hydraulic head variance computed by our perturbed moment equations solved with the technique of random domain decomposition and by numerical Monte Carlo for some degrees of uncertainty in the layering location in the case of mean flow perpendicular to the layering. The maximum discrepancy between the two solutions is about $2 \%$. A similar quality of agreement was observed in comparing all significant moments of hydraulic head and flux, therefore, we do not compare them further.
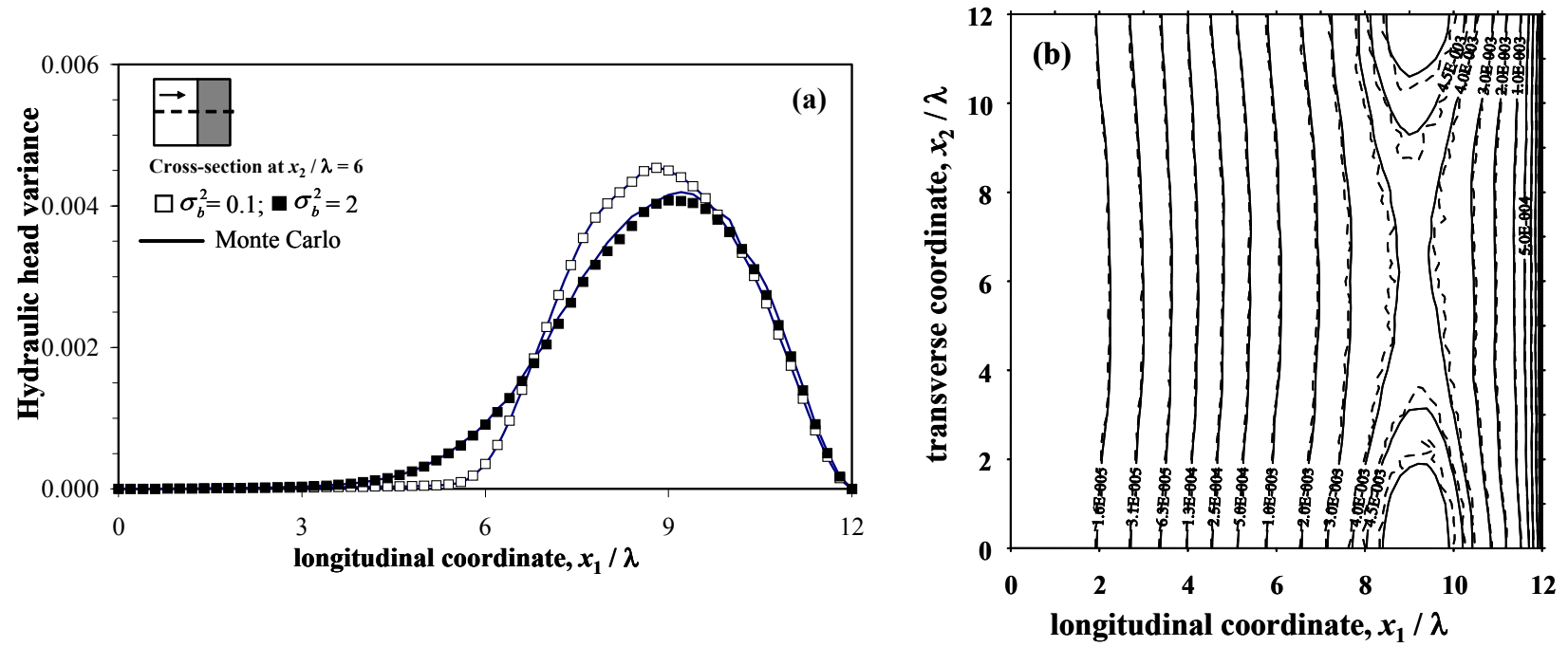

Figure 2. Perpendicular Flow. Comparison between hydraulic head variance computed by numerical Monte Carlo simulations and moment equations with RDD: (a) cross-section $x_{2} / \lambda=$ 6 for $\sigma_{b}^{2}=0.1,2$; (b) contour map for $\sigma_{b}^{2}=2$ (dashed: Monte Carlo; solid: Moment Equations).

\section{Ensemble moments of hydraulic head}

\subsection{Mean hydraulic head}

In the following we examine the behavior of mean hydraulic heads along cross-sections located at the midpoint of the flow domain and normal to the layering. When mean flow develops parallel to the stratification, profiles of mean hydraulic head decrease linearly from left $\left(x_{1} / \lambda=0, \mathrm{H}_{\mathrm{A}}=1.6\right)$ to right $\left(x_{1} / \lambda=12, \mathrm{H}_{\mathrm{B}}=1.0\right)$ independently of the location of the 
internal boundary. In the case of mean flow normal to stratification, Figure 3 a depicts profiles of conditional mean hydraulic head along the transverse cross-section $x_{2} / \lambda=6$ and three realizations of the location of the internal boundary, $b / \lambda=x_{2} / \lambda=3,6,9$, together with the mean hydraulic head resulting from the homogeneous approximation. The perfectly known geometry of the layered system corresponds to $\sigma_{b}^{2}=0$. As noted before, head distributions obtained from Monte Carlo simulations and from our moments equations are indistinguishable. Hence only the moments equation solution is represented in all our examples. Figure $3 \mathrm{~b}$ shows the distribution of mean hydraulic head, along the transverse cross-section $x_{2} / \lambda=6$ for several values of $\sigma_{b}^{2}$. The effect of uncertainty in the internal boundary on one's ability to estimate hydraulic head is apparent. As $\sigma_{b}^{2}$ increases, the profile of mean head distribution is smoothed out and the sharp discontinuity at the interface of the two materials vanishes. In this case, modelling the heterogeneous medium by introducing only randomness in the location of the layering and considering medium properties as deterministic renders a pattern which is very similar to that of our composite model, with slightly steeper gradients at locations $x_{1} / \lambda>6$ (not shown). Analogously, adopting a linear trend model for the hydraulic conductivity yields a pattern which is similar to the homogeneous approximation (not shown). We also note that the scenario corresponding to $\sigma_{b}^{2}$ $=0$ can be interpreted as the result of representing the spatial distribution of hydraulic conductivity by a step function trend model. In practice, adopting this type of trend function requires some information about the distribution of materials within the flow domain. However, even such a trend model renders results which are qualitatively and quantitatively different from those of a full composite medium scheme.
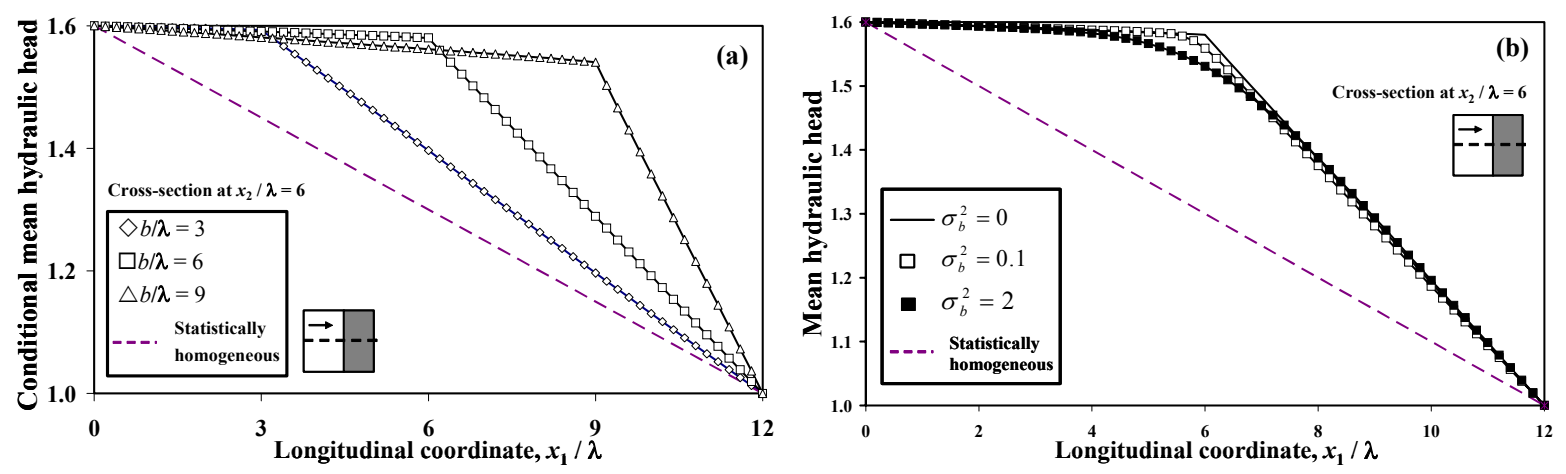

Figure 3. Perpendicular flow. Cross-section $x_{2} / \lambda=6$ of (a) conditional mean hydraulic head for several realizations of $b$ and (b) mean hydraulic head for various levels of uncertainty of $b$.

\subsection{Variance of hydraulic head}

In the following we discuss the behavior of the effect of boundary uncertainty on solutions for head variance. Figure $4 \mathrm{a}$ depicts the conditional hydraulic head variance along the median transverse cross-section at $x_{1} / \lambda=6$ for mean flow parallel to the layering and three realizations of the location of the internal boundary, $b / \lambda=x_{2} / \lambda=3,6$, 9. The corresponding depiction for mean flow normal to the layering is shown in Figure $4 \mathrm{~b}$. The distribution of hydraulic head variance corresponding to a statistically homogeneous medium with unit variance of $Y$ is also reported. Figure 5a shows profiles of total head variance as computed by our moment equations, homogeneous approximation and the approximation replacing the statistically inhomogeneous conductivity field by a pseudo-homogeneous random field with a deterministic linear trend. The corresponding depiction for mean flow normal to the layering is offered in Figure 5b. 
Although the internal boundary obviously affects each realization, the homogeneous approximation generally under-estimates head variance and actually reduces head variance in the vicinity of the boundary where in reality variance increases (Figure 4a). Head variances derived from the composite medium approach show a similar internal boundary effect, although it is smoothed by uncertainty about the boundary location (Figure 5a). For the three $\sigma_{b}^{2}$, head variance is slightly lower in the less permeable material than is variance in the more permeable material. The nature of this behavior is explained by the structure and the functional dependencies evidenced in the moment equations. Increasing the variance of $b$ causes a reduction of the hydraulic head variance at points occupied by the more conductive material in the flow scenario corresponding to the mean location of the internal boundary (Figure $5 \mathrm{a}, \sigma_{b}^{2}=0$ ). Conversely, hydraulic head variance increases at points occupied by the less conductive material in the flow scenario corresponding to $\sigma_{b}^{2}=0$. The effect of introducing variability of $b$ corresponds to hydraulic head variance at a point to be evaluated by the weighting process (5). Therefore, when compared to the scenario $\sigma_{b}^{2}=0$, any variance of $b$ will cause appearance of high (low) conductivities at points corresponding to low (high) conductivities for zero variance of $b$, thus impacting the results of the weighting procedure. Accounting for increasing uncertainty in the location of the internal boundary causes an ever increasing interference of the effects of the two types of materials.

Once again, the homogeneous case is qualitatively different, showing a minimum in variance at the midpoint of the transverse cross section. Superimposing a (deterministic) linear trend to the heterogeneous hydraulic conductivity field results in a distribution of head variance which is qualitatively similar to the results of the homogeneous approximation, but with considerably lower values. On the other hand, modelling the heterogeneous medium by introducing randomness only in the location of the layering and characterizing each material by constant properties results in a zero-variance hydraulic head field. This is so because the hydraulic head for each realization of $b$ is a straight line, as discussed in the previous subsection.

Mean flow perpendicular to the layering also illustrates the general inadequacy of (pseudo) homogeneous approximations. In this flow scenario, conditional hydraulic head variance (Figure 5b) is generally much lower in the more conductive subdomain than in the less conductive. This behavior can be explained by the fact that pressure can equilibrate more easily where flow is least restricted. In fact, on the basis of the Monte Carlo results and Figure $3 \mathrm{a}$ we note that hydraulic heads display lower gradients in the more conductive material, thus allowing for lower variations of $h(\boldsymbol{x})$ in the space of realizations of $Y$.

When considering the composite medium model (Figure $5 b$ ) we notice that increasing the variance of $b$ causes a reduction of the hydraulic head variance at points occupied by the less conductive material in the flow scenario corresponding to the mean location of the internal boundary (Figure $5 \mathrm{~b}, \sigma_{b}^{2}=0$ ). Conversely, hydraulic head variance increases at points occupied by the more conductive material in the flow scenario corresponding to $\sigma_{b}^{2}=$ 0 . This is explained again by the fact that for each realization in the space of $b$, variability of hydraulic head within the more conductive material is considerably less than that occurring in the less conductive medium. Therefore, the uncertainty in the location of the internal boundary causes an interference of the effects of the two types of materials and produces an overall result which tends to a configuration qualitatively similar to that of a homogeneous approximation. For the same physical reason, introducing a linear trend in $Y$ renders a variance similar to that of the homogeneous approximation, but skewed toward locations occupied by the less conductive material. 

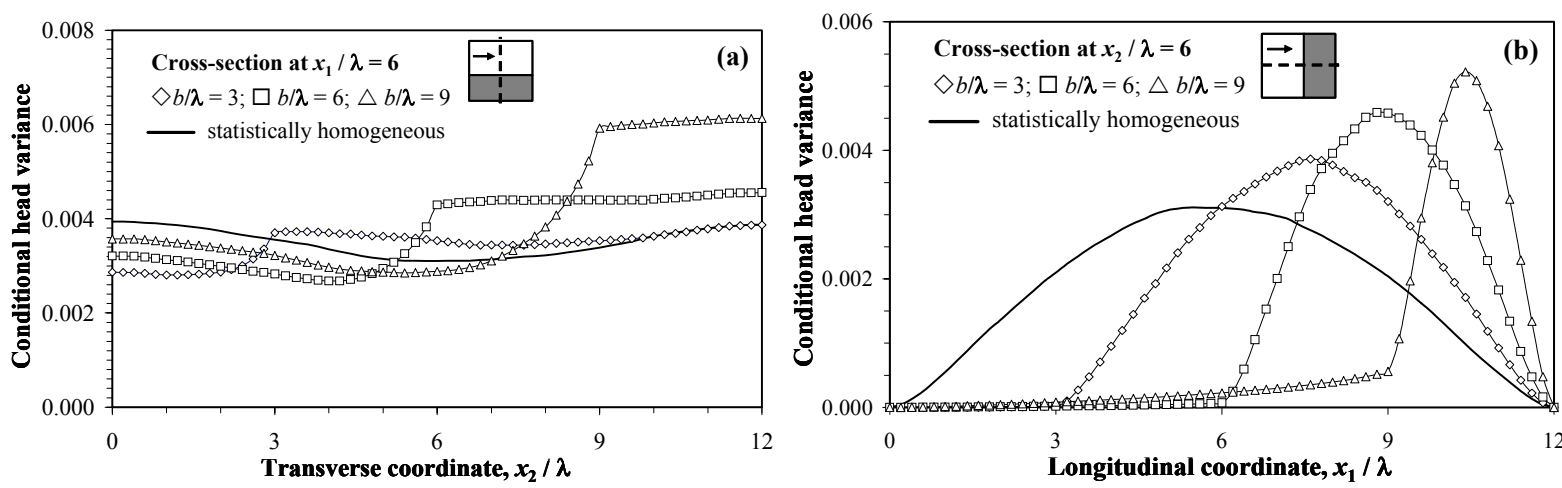

Figure 4. Cross-sections of conditional distributions of hydraulic head variance compared to the homogeneous approximation for (a) parallel and (b) perpendicular flow.
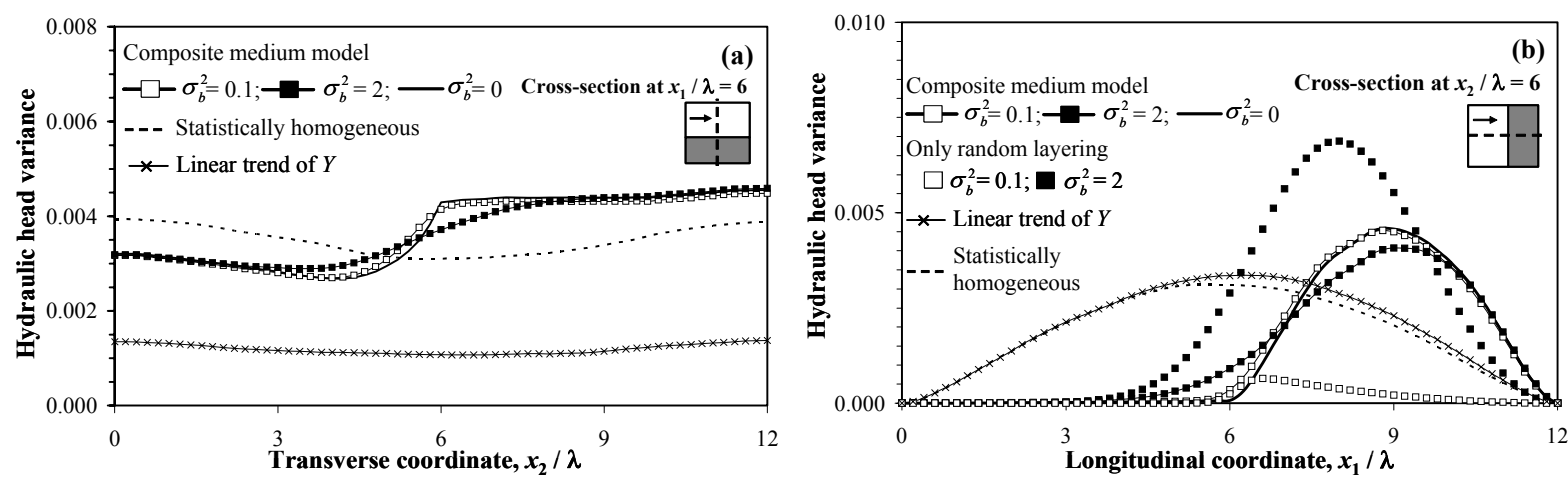

Figure 5. Cross-section $x_{1} / \lambda=6$ of hydraulic head variance computed by the composite medium model, homogeneous approximation, and imposing a linear trend in the log conductivity for (a) parallel and (b) perpendicular flow.

The model prescribing only randomness in layering location renders profiles with shape similar to the composite medium model, but qualitatively and quantitatively dissimilar from the latter. Specifically, there is a continuous increase of hydraulic head variance with $\sigma_{b}^{2}$. This behavior is imputable to the adopted Normal distribution of $b$ and descends straightforwardly from the results of Figure $3 \mathrm{a}$. In the ensemble of realizations of $b$, the weight of realizations that are symmetric with respect to the mean layering location is the same. However, realizations where the less conductive material extends over a larger portion of the domain always result in a larger contribution to the overall variance of the process. Where for small variance of $b$ only a few realizations of the internal boundary (specifically those concentrated around the mean layering location) affect the statistical averaging process, the number of relevant (statistically meaningful) realizations increases with the variance of $b$. When $\sigma_{b}^{2}$ is small, evaluating hydraulic head variance upon considering uncertainty only in the layers location renders results which underestimate those offered by a composite medium model. The opposite occurs for larger variances of $b$. This is a direct consequence of the weighting process in the ensemble of realizations of $b$ and descends from the results of Figure $4 \mathrm{~b}$ for the composite medium model (where we see that the profiles at $b / \lambda=3,9$ have the same weight but remarkably different contribution to the global (weighted) variance of the process (8)) and Figure 3a for the model which considers only randomness in the layering location.

\section{Ensemble moments of flux}


Since mean transverse flux is always zero and longitudinal mean flux does not show any particular feature, we will concentrate only on the discussion of flux variance. The latter is particularly relevant in the context of solute transport in layered formations.

\subsection{Variance of flux}

Figure $6 \mathrm{a}$ and $6 \mathrm{~b}$ depict profiles of variance of transverse flux, $\sigma_{q_{2}}^{2}(\boldsymbol{x})$, computed by the composite medium model, homogeneous approximation, and imposing a linear trend in the log conductivity for mean flow respectively parallel and perpendicular to layering. For reference, we have reported the analytical solution derived by Rubin [1990], $\sigma_{q_{2}}^{2}=U \sigma_{Y}^{2} / 8$ (where $U$ is mean longitudinal flux and $\sigma_{Y}^{2}=1$ ), for two-dimensional mean uniform flow in an infinite domain. Results from the homogeneous approximation and linear theory of Rubin have been evaluated upon considering a statistically homogeneous domain with unit $\sigma_{Y}^{2}$ and $\langle Y\rangle=3.5$, or 7.0 and are in excellent agreement, considering the limited size of the domain.
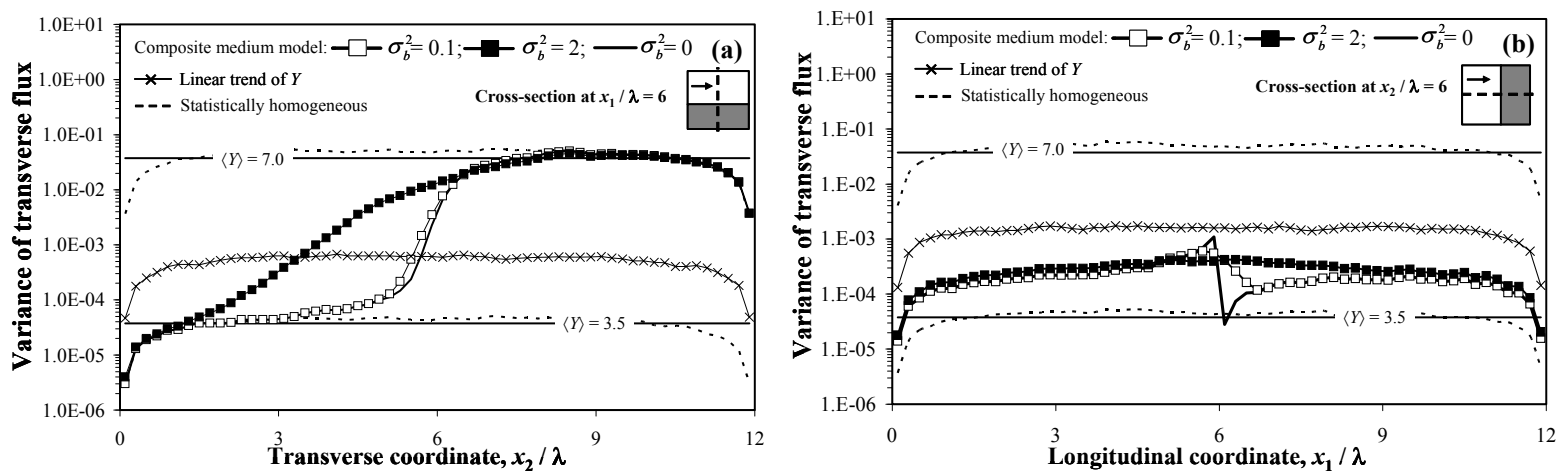

Figure 6. Cross-sections of variance of transverse flux, computed by the composite medium model, homogeneous approximation, and imposing a linear trend in the log conductivity for (a) parallel and (b) perpendicular flow. The horizontal solid lines correspond to the linear approximation of Rubin [1990] for an infinite medium composed by one of the two materials.

Use of a model relying on unknown geometry but constant hydraulic properties renders a zero-variance transverse flux in both flow configurations, therefore affecting significantly evaluation of contaminant lateral spreading. Imposing a linear trend on hydraulic conductivities yields results which are intermediate to those given by employing a homogeneous approximation of the conductivity field. The effect of the composite medium model is apparent from the Figures and qualitatively different from those relying on (pseudo) homogeneous approximations.

The corresponding profiles of longitudinal flux variance are depicted in Figure 7 for flow parallel to layering. In this case it is interesting to point out the behavior of the solution based on imposing randomness only on $b$. Variance of longitudinal flux peaks at the center of the domain and tends to zero toward the horizontal boundaries. The portion of the domain within which it remains nonzero increases with $\sigma_{b}^{2}$, eventually covering the whole domain. This behavior is explained by arguments analogous to those previously described for hydraulic head variance. In the case of mean flow perpendicular to layering (not shown) the general qualitative behavior of the different models is similar to that presented in Figure 7. However, in this case the model relying on unknown geometry and constant properties renders a 
constant value of longitudinal flux variance throughout the entire domain. Again, this is related to reasons explained in previous sections and to mass conservation arguments.

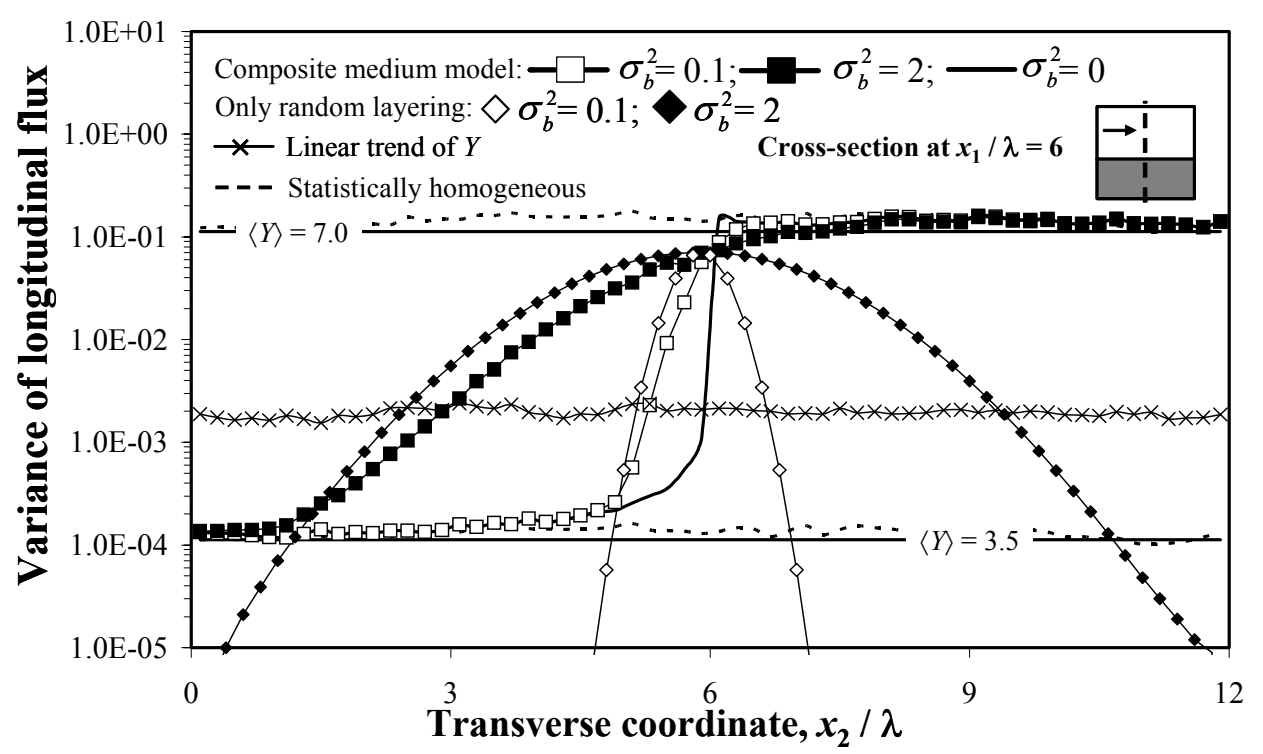

Figure 7. Parallel Flow: cross-section $x_{1} / \lambda=6$ of variance of longitudinal flux computed by the composite medium model, homogeneous approximation, uncertain geometry but known conductivities, and imposing a linear trend in the log conductivity. The horizontal solid lines correspond to the linear approximation of Rubin [1990] for an infinite medium composed by one of the two materials.

\section{Conclusions}

Our work leads to the following major conclusions:

1. The random composite media approach, which employs random domain decomposition in the context of groundwater flow moment equations, allows one to explicitly take into account the separate effects of material and geometric uncertainty on statistical moments of hydraulic head and flux.

2. Our examples, based on two-dimensional flow in a two-material layered heterogeneous medium emphasize the qualitative and quantitative unsuitability of the homogeneous approximation for mean flow parallel to the layering and then for mean flow normal to the layering. In practice, these models allow describing flow parallel and tilted (e.g., due to recharge) to aquifer stratification in natural sedimentary formations.

3. Modeling the essentially inhomogeneous conductivity field with pseudo-homogeneous random fields based on a linear trend of the conductivities of the two contrasting materials yields misleading predictions of (ensemble) moments of hydraulic head and flux.

4. Use of a step function trend model for hydraulic conductivity distribution might improve the overall quality of the agreement with moments computed via the composite medium scheme. However: (a) from a practical standpoint adopting this type of trend function always requires some information about the distribution of materials within the flow domain; (b) even such a trend model renders results which are qualitatively and quantitatively different from those of a full composite medium scheme.

5. We have analyzed the relative importance of uncertain geometry and uncertain conductivity by comparing the special case in which the layers geometry is random, 
but the hydrogeologic properties of each material are fixed. Disregarding properties variability within materials leads to incorrect description of the ensemble behavior of the system. Notable cases are offered by (a) the zero-variance hydraulic heads in the case of mean flow parallel to stratification and (b) the resulting zero-variance transverse component of flux for mean flow parallel and perpendicular to stratification. The latter can be critical factors in risk assessment practice dealing with quantitative and qualitative aspects of groundwater.

6. Stochastic hydrogeology has been held back by its inability to accommodate nonstationary conductivity fields. Geophysical and geological characterization techniques have reached a point where it is now possible to outline material facies with some uncertainty about their boundaries and internal properties like conductivity (see Rubin et al., 1998 for a review). When such data is available, RDD reduces uncertainty in head and flux estimates by exploiting all available information. In particular, it represents macroscopic uncertainty about the extent and location of facies in terms of geometry (rather than as a trend, for instance), which is the natural representation of randomness at the facies scale. The RDD model is broad enough to include most natural porous media, while allowing for obvious simplifications when one scale of uncertainty dominates the other.

\section{Acknowledgements}

This work was performed under the auspices of the U.S. Department of Energy (DOE): DOE/BES (Bureau of Energy Sciences) Program in the Applied Mathematical Sciences contract KC-07-01-01 and Los Alamos National Laboratory under LDRD 98604. This work made use of STC shared experimental facilities supported by the National Science Foundation under Agreement No. EAR-9876800. This work was supported in part by the European Commission under Contract No. EVK1-CT-1999-00041 (W-SAHaRA).

\section{References}

Desbarats, A. J., 1990: Macrodispersion in sand-shale sequence. Water Resour. Res., 26(1), $153-163$.

Guadagnini, A., and S. P. Neuman, 1999a: Nonlocal and localized analyses of conditional mean steady state flow in bounded, randomly nonuniform domains, 1 . Theory and computational approach. Water Resour. Res., 35, 2999 - 3018.

Guadagnini, A. and S. P. Neuman, 1999b: Nonlocal and localized analyses of conditional mean steady state flow in bounded, randomly nonuniform domains, 2, computational examples, Water Resour. Res., 35 (10), 3019-3039.

McLaughlin, D. and E. F. Wood, 1988: A distributed parameter approach for evaluating the accuracy of groundwater model predictions, 1. Theory. Water Resour. Res., 24(7), $1037-1047$.

Rubin, Y, S. S. Hubbard, A. Wilson, and Mark A. Cushey, 1998: Aquifer characterization, Handbook of Groundwater Hydrology, CRC Press, 10.1-10.33.

Winter, C. L., and D. M. Tartakovsky, 2000: Mean flow in composite porous media. Geophys. Res. Lett., 27, 1759 - 1762.

Winter, C. L., and D. M. Tartakovsky, 2002: Groundwater flow in heterogeneous composite aquifers. Water Resour. Res., 38(8), doi:10.1029/2001WR000450.

Winter, C. L., D. M. Tartakovsky, and A. Guadagnini, 2002: Numerical solutions of moment equations for flow in heterogeneous composite aquifers. Water Resour. Res., 38(5), doi: 10.1029/2001WR000222.

Winter, C.L., D.M. Tartakovsky and A. Guadagnini, 2003: Moment equations for flow in highly heterogeneous porous media, Surveys in Geophysics, 24, 81 - 106. 